\title{
«La vraie nature du tragique ». Norma et le classicisme européen à l'âge romantique
}

\section{Luca Zoppelli}

\section{(2) OpenEdition}

1 Journals

Édition électronique

URL : http://journals.openedition.org/edl/1149

DOI : $10.4000 /$ edl. 1149

ISSN : 2296-5084

Éditeur

Université de Lausanne

\section{Édition imprimée}

Date de publication : 15 décembre 2016

Pagination : $33-48$

ISBN : ISBN 978-2-940331-50-5

ISSN : 0014-2026

\section{Référence électronique}

Luca Zoppelli, « "La vraie nature du tragique ». Norma et le classicisme européen à l'âge romantique », Études de lettres [En ligne], 4 | 2016, mis en ligne le 15 décembre 2019, consulté le 16 décembre 2020. URL : http://journals.openedition.org/edl/1149; DOI : https://doi.org/10.4000/edl.1149 


\section{"LA VRAIE NATURE DU TRAGIQUE». NORMA ET LE CLASSICISME EUROPÉEN À L'ÂGE ROMANTIQUE}

L'article propose une analyse philosophique, esthétique et musicale de Norma de Bellini. Il traite de la manière dont le compositeur et son librettiste, Felice Romani, ont construit le sens du "tragique» dans l'opéra, considéré, selon une conception romantique, comme une forme élevée de représentation de l'individu, de ses agissements et des conséquences qu'il doit endurer. L'auteur analyse dans ce sens notamment deux scènes de l'opéra: le duo Norma - Adalgisa à la fin du premier acte et le concertato qui clôt l'œuvre.

Dans le chapitre XXXVII des suppléments au Monde comme volonté et comme représentation, son chef-d'œuvre philosophique, Arthur Schopenhauer s'occupe de théâtre: il cherche un exemple parfaitement accompli de l'efficacité de la catastrophe tragique. Etonnement, il ne cite ni Shakespeare, auteur qu'il admire pourtant, ni Schiller, ni les tragiques grecs. L'exemple choisi est Qual cor tradisti, la section du finale II de Norma suivant l'auto-dénonciation de la protagoniste, dans lequel Schopenhauer lit l'apaisement de la volonté de vivre et l'acceptation du destin. Dans l'opéra de Felice Romani et de Vincenzo Bellini, on trouverait d'ailleurs: "Un vrai modèle de combinaison tragique des motifs, de progression tragique de l'action, de développement tragique» - tout ce qui, en somme, fait «la vraie nature du tragique» ${ }^{1}$.

I. A. Schopenhauer, Die Welt als Wille und Vorstellung, Ergänzungen zum dritten Buch, Kapitel 37 (Vol. II, p. 496): "Hier sei es erwähnt, daß selten die ächt tragische Wirkung der Katastrophe, also die durch sie herbeigeführte Resignation und 
Certes, Schopenhauer parle en philosophe plus qu'en théoricien du théâtre. Son but est de montrer que la catastrophe tragique nous soustrait à l'empire de la "volonté", la force vitale aveugle qui œuvre au fond de tout être vivant et qui le condamne à la souffrance perpétuelle. Toutefois, cette consécration de Norma comme «tragédie parfaitement accomplie» saisit également son noyau esthétique, la volonté d'atteindre un vrai classicisme tragique, opposé au caractère simplement funeste de la plupart de la production seria de l'âge romantique.

Le problème d'une définition correcte du "tragique» était au cœur des réflexions de l'époque, même en marge d'un spectacle d'opéra. Dans l'usage linguistique courant (plus que jamais aujourd'hui), l'adjectif «tragique " a glissé vers un synonyme de "calamiteux, funeste». L'effort théorique qui réunissait les intellectuels européens autour de 1800 visait, en revanche, à en limiter l'emploi, en l'utilisant comme forme particulière élevée - de représentation de l'individu, de ses agissements et des conséquences qu'il doit endurer. Haut placé, ce protagoniste ne devra pas succomber à l'œuvre accidentelle du hasard ni à la violence arbitraire d'un ennemi plus puissant (déjà pour Aristote, l'hostilité entre ennemis ou la mort accidentelle n'était pas un ressort tragique adéquat). La représentation de l'injustice, des rapports asymétriques de pouvoir, des malheurs qui dérivent de mobiles matériel ou quotidien est réservée aux nouveaux genres dramatiques bourgeois, comme le drame ou le mélo: la vraie tragédie doit s'abstenir d'y recourir. L'échec du personnage est censé dériver, d'une manière logique et inévitable, de la nature irréconciliable des contradictions qui se situent soit dans son intériorité, soit dans le système de valeurs de la constellation de personnages à laquelle il appartient. Les situations se cristallisent sous la forme d'affrontements entre tendances

Geisteserhebung der Helden, so rein motivirt und deutlich ausgesprochen hervortritt, wie in der Oper Norma, wo sie eintritt in dem Duett "Qual cor tradisti, qual cor perdesti", in welchem die Umwendung des Willens durch die plötzlich eintretende Ruhe der Musik deutlich bezeichnet wird. Ueberhaupt ist dieses Stück - ganz abgesehen von seiner vortrefflichen Musik, wie auch andererseits von der Diktion, welche nur die eines Operntextes seyn darf - und allein seinen Motiven und seiner innern Oekonomie nach betrachtet, ein höchst vollkommenes Trauerspiel, ein wahres Muster tragischer Anlage der Motive, tragischer Fortschreitung der Handlung und tragischer Entwickelung, zusammt der über die Welt erhebenden Wirkung dieser auf die Gesinnung der Helden, welche dann auch auf den Zuschauer übergeht: ja, die hier erreichte Wirkung ist um so unverfänglicher und für das wahre Wesen des Trauerspiels bezeichnender, als keine Christen, noch Christliche Gesinnungen darin vorkommen ». 
et principes souvent nobles et légitimes, dont toutefois l'enchevêtrement génère une radicalisation paradoxale qui entraîne l'issue funeste (voir à cet égard l'analyse d'Antigone menée par Hegel dans son Esthétique). La "vraie» tragédie compose un système de conflits abstraits et se refuse de distribuer les personnages sur un modèle axiologique des «bons et des méchants». Le protagoniste ne peut pas être entièrement innocent, ses antagonistes ne peuvent non plus être présentés comme de simples agents de la contrainte, de la persécution. En outre, le conflit a un caractère dialectique, fondé non pas sur la contrainte a priori que représente l'inégalité de pouvoir, mais sur un échange entre pairs ou sur le conflit intérieur qui pose le personnage face à une série de dilemmes argumentatifs. Selon Marc Fumaroli, les monologues de tragédies ne sont souvent que "des délibérations où les différentes personae possibles du même personnage sont tour à tour évoquées par lui-même " ${ }^{2}$. D'où le caractère logocentrique de la vraie tragédie - le fait que les personnages se présentent et argumentent à la première personne - et le rôle limité des éléments gestuels et visuels. En principe, le héros de la tragédie est, à tout moment du parcours, libre et responsable de ses actes: sa chute en découle, mais le fait d'assumer librement les conséquences a souvent la valeur de rachat moral. D'où l'interprétation du destin tragique, dans la théorisation philosophique de l'idéalisme allemand (Schiller notamment), comme forme paradoxale et extrême de liberté: l'on triomphe de la nature aveugle par la libre acceptation du sort. En revanche, l'univers du "drame" adopte une perspective axiologique, nette et tranchante. Il doit y avoir des victimes et des oppresseurs (éventuellement, dans une phase ultérieure, des personnages qui sont tantôt victimes de certains, tantôt oppresseurs d'autres, comme dans le théâtre d'Hugo dans lequel les fonctions restent toutefois nettement définies). La neutralisation de la perspective morale prônée par la tragédie classique - dans laquelle chaque intervenant peut réclamer un moment de légitimité pour son action - est intenable: comme l'écrit Alessandro Manzoni, deux tendances opposées ne peuvent être bonnes et justes toutes les deux ${ }^{3}$. Dans la tirade finale de sa dernière tragédie, Adelchi (1822), Manzoni met dans la bouche de son protagoniste, mourant et désabusé, une maxime qui résume bien cette attitude: sol non resta / che far torto, o patirlo («Il ne reste plus qu’à faire

2. M. Fumaroli, Héros et orateurs, p. 311.

3. A. Manzoni, Materiali estetici, p. 503. 
le mal, ou à le subir", Acte $V$, scène 8 , v. 353-354) ${ }^{4}$. Dans cette amère constatation existentielle, nous reconnaissons également un principe d'organisation dramaturgique puisqu'il implique une distinction entre personnages libres et non libres, actifs et passifs, entre un discours du pouvoir qui se communique et une impuissance condamnée au silence. Conscient de la difficulté que pose l'application de tels principes au genre de la tragédie, Manzoni l'abandonne et se consacre au roman.

Quelques années plus tard, Richard Wagner - sans connaître le passage de Schopenhauer (il n'en découvrira les écrits que dans le courant des années 1850) - porte sur Norma un regard semblable. Dans une série d'articles et comptes rendus publiés en 1837, il affirme que le poème, chose rare pour un livret d'opéra, parvient à atteindre le niveau tragique du théâtre de la Grèce classique et à souligner le caractère solennel du tout, ainsi que la noblesse de la musique de Bellini :

L'action, qui évite tout coup de théâtre et tout effet facile, rappelle involontairement les modes de la tragédie grecque; peut-être l'idée avancée par Schiller dans sa préface à La fiancée de Messina - que l'opéra puisse faire revivre sur nos scènes la tragédie de l'antiquité serait confirmée à l'écoute de cette Norma 5 .

En effet, le type tragique qui fonde le nœud dramatique sur les contradictions internes des personnages est ici parfaitement accompli. Norma (grâce en partie à la composition de la compagnie de chanteurs pour laquelle elle est conçue) est l'un des rares exemples d'opéra italien de l'époque qui échappe à l'intrigue typique fondée sur un couple d'amoureux soprano/ténor, dont l'union est empêchée par une figure tyrannique et odieuse de baryton. Ici, sur le plan actanciel abstrait, c'est Norma qui fonctionne comme antagoniste du couple d'amoureux Adalgisa/Pollione; elle le fait toutefois en pleine légitimité morale, alors que le comportement de Pollione, qui envisage d'abandonner la mère de ses enfants, est très douteux. Cependant, Norma est coupable sur

4. A. Manzoni, Adelchi, p. 130.

5. «Die Handlung, die aller Theatercoups u. blendender Effekte entbehrt, erinnert unwillkürlich an die Haltung der griechischen Tragödie, u. vielleicht möchte Schillers Einsicht, die er in die Vorrede zu seiner Braut von Messina aussprach, dass er von der Oper Alles für die Wiedererscheinung der antiken Tragödie auf unserer Bühne gehofft habe, durch eine Anhörung dieser Norma wieder neue Nahrung erhalten haben" (R. Wagner, Bellini, p. 7). 
d'autres plans: celui de la loyauté politique envers son peuple oppressé (l'amour pour Pollione lui a toujours fait différer l'instant où elle donnera, en prêtresse et interprète de la volonté des dieux, le signal de la révolte contre les Romains) et celui de l'amour maternel, puisqu'elle éprouve pour un instant, comme Médée, l'envie d'égorger ses enfants (une faute inhumaine, "oltre ogni umana idea", comme elle le dit). Quant à Pollione, il se rachète dans les scènes finales par son attitude héroïque et désintéressée.

En écrivant que cette structure de type classique se serait déterminée «involontairement», Wagner faisait fausse route. Felice Romani, librettiste de très grande qualité mais également critique littéraire, journaliste et savant spécialisé dans l'étude de la mythologie, était un adepte de l'esthétique classique. En tant que librettiste, il a certes été obligé de multiplier les compromis avec le goût de son public (notamment celui de Milan, qui suivait avec engouement l'évolution du drame français vers le romantisme frénétique), la volonté des interprètes et les préférences des compositeurs. Il n'a pourtant jamais renoncé à régulariser et à atténuer ses sources dans la direction classique. Bellini, à son tour, avait probablement assimilé certains préceptes classiques auprès du milieu napolitain dans lequel il s'était formé. Dans les années suivantes, lorsqu'il est installé à Paris, ses lettres semblent faire écho à ces notions, comme à la nécessité d'éviter les personnages trop «méchants» et de ne pas en faire la source des malheurs des protagonistes ${ }^{6}$. A propos du personnage de Norma, il apprécie le jeu tragique de Giuditta Pasta, pour qui le rôle est conçu, et en qui le public de l'époque voit un rempart de l'esthétique classique contre le déferlement romantique. En revanche, Bellini considère que Giulia Grisi, excellente cantatrice par ailleurs, n'est pas adaptée à chanter Norma parce qu'elle ne peut comprendre ni ressentir les personnages de type élevé (i caratteri elevati non li capisce, né li sente). Elle ne posséderait ni l'instinct, ni l'instruction nécessaires pour les représenter avec la noblesse et la hauteur de style que ces rôles exigent (con quella nobiltà ed alto stile che richiedono) 7 .

6. Voir L. Zoppelli, «Il personaggio belliniano».

7. Lettre à Francesco Florimo, 1 juillet 1835 (C. Neri, Vincenzo Bellini, p. 429). 
La source de l'opéra, la tragédie d'Alexandre Soumet ${ }^{8}$, unissait le motif de l'infanticide, associé surtout au mythe de Médée, à celui, fréquent dans l'opéra européen depuis l'âge des Lumières, de la vestale/ prêtresse qui enfreint ses vœux. Le cadre celtique mis à la mode, entre autres, par Les Martyrs de Chateaubriand, l'influence ossianique, l'élément chrétien et le recours à des techniques dramatiques empruntées aux théâtres de boulevard et aux codes du drame s'y ajoutent ${ }^{9}$. Soumet, qui était lié aux cercles romantiques, visait en fait une espèce de conciliation entre la tradition tragique et l'esthétique du romantisme, une position qui lui assura beaucoup de respect pendant un certain temps. En 1846, Théophile Gautier jugeait encore Norma comme «une œuvre d'inspiration originale, et peut-être la dernière des tragédies possibles de notre temps ${ }^{10}$. A la longue, toutefois, cette ambigüité nuisit à l'image de Soumet, resté (toujours selon Gautier) «romantique pour les classiques et classique pour les romantiques" ${ }^{11}$, et vite oublié. Or, c'est bien du côté romantique de cette dramaturgie que l'œuvre culmine se terminant par l'infanticide et la folie de la protagoniste - une folie, qui plus est, étalée sur scène, véritable "entorse aux usages classiques" ${ }^{12}$. Romani et Bellini laissent tomber, parmi d'autres caractéristiques de la pièce, ce dénouement frénétique. Ils le font peut-être pour des raisons d'opportunité théâtrale (ne pas obliger Pasta à réitérer la scène d'aliénation mentale par laquelle se terminait Anna Bolena de Gaetano Donizetti, présenté à Milan un an auparavant ${ }^{13}$ ), mais le résultat renforce, bien évidemment, le caractère tragique et classique de l'œuvre. A cette époque, le topos de la folie - la représentation d'un personnage qui perd la raison sous la pression des évènements et de l'environnement social est le signe d'une esthétique du drame ou d'une condition sociale non

8. Paris, Odéon, 6 avril 1831. Huit mois auront donc suffi pour que le texte parvienne à Milan et que Romani en tire un livret, mis en musique par Bellini et que le spectacle soit monté à la Scala (première le 26 décembre).

9. Voir Ch. Cazaux, "Alexandre Soumet, Felice Romani»; D. Colas, «Aux sources du personnage de Norma"; M. Melai, Les derniers feux de la tragédie classique au temps du romantisme, p. 52 sq., 177-180.

Io. Feuilleton dans La presse, republié dans Th. Gautier, Histoire de l'art dramatique en France depuis vingt-cinq ans, p. 227.

II. Th. Gautier, Histoire du romantisme, p. 187.

I2. D. Colas, «Aux sources du personnage de Norma», p. 34.

13. Voir P. Cecchi, "Temi letterari e individuazione melodrammatica in Norma di Vincenzo Bellini». 
élevée. Dans Il Pirata, leur première collaboration, Romani et Bellini avaient épousé l'esthétique du mélodrame qui caractérisait leur source théâtrale (Bertram ou le Pirate du baron Taylor), ce qui justifiait la folie d'Imogene; le somnambulisme d'Amina ou la folie d'Elvira (I Puritani) s'expliquent par la condition sociale des protagonistes et par les codes du genre (un drame sentimental et villageois dans le premier cas, historique et bourgeois dans le second). Même dans Anna Bolena - toujours un livret de Romani - la déraison d'Anna peut s'expliquer par la nature du personnage, femme de condition sociale relativement simple jadis, élevée au trône par le caprice du roi (et dont le dérangement s'exprime surtout par une régression nostalgique à un état d'esprit enfantin et à une condition plus humble et privée). Dans les Mémoires de deux jeunes mariées de Balzac, achevés en 1841, Renée de L'Estorade, très attachée aux valeurs aristocratiques, affirme que «les gens d'esprit ne deviennent pas fous comme les bourgeois ou comme les sots» ${ }^{14}$. En vrai personnage tragique de type élevé, la Norma de l'opéra ne devient pas folle "comme une bourgeoise». Certes, elle traverse un parcours qui la mène au bord de l'abîme, permettant à Bellini de mettre à contribution les techniques le plus «romantiques» dont il dispose, mais elle parvient à récupérer la maîtrise de soi, l'auto-détermination et le contrôle linguistique propres au personnage tragique et accepte noblement l'issue de la situation intenable provoquée par ses «fautes» morales.

Ce parcours de crise et de réappropriation de la nature tragique du personnage débute par une cavatine, Casta Diva che inargenti, dont le texte consiste en une prière pour la paix adressée à la lune - un souhait qui, dans l'âme de Norma, unie secrètement à un ennemi de son peuple et craignant de le perdre, a des résonances personnelles et intimes. Cela se passe dans le cadre d'une cérémonie collective, en présence de tout le peuple. Il s'agit par conséquent d'une confession intime structurée selon la nature communicative d'un acte rituel et qui s'adresse à un interlocuteur fictif, la lune. L'apostrophe à l'astre nocturne est un topos qui revit avec force dans la culture italienne du début du siècle: pour le lecteur d'aujourd'hui, il est notamment incarné par l'œuvre de Giacomo Leopardi qui s'en sert souvent. Ce qui nous intéresse ici est moins le rôle d'ambiance, typique du premier romantisme européen, assurée par le thème de la lune, que la fonction grammaticale que l'astre ne cesse

I4. H. de Balzac, La Comédie humaine, I, p. 403. 
d'incarner dans ces textes, celle de l'objet silencieux auquel le "je» lyrique s'adresse. Grâce à ce topos, Romani nous présente Norma dans une situation d'énonciation structurée et contrôlée, très classique donc, malgré la dimension personnelle et privée du message. Sorte de mise à jour de la confidente de tragédie racinienne, la lune permet à Norma de nous livrer non pas un monologue fragmenté, erratique et silencieux, typique des personnages humbles et "passifs", dont la nature émotionnelle serait saisie et dévoilée par le compositeur en narrateur omniscient, mais une confession organisée en forme strophique dont elle semble garder la pleine responsabilité autoriale. Loin de déléguer l'expression de soi à des moyens externes, narratifs, gestuels, le personnage s'incarne dans la seule parole chantée, par une démarche d'auto-stylisation classique. Après le seuil phatique d'une longue ritournelle instrumentale d'introduction, la ligne vocale assume la primauté ininterrompue d'une mélodie «monodirectionnelle» qui se renouvelle sans cesse, évitant le plus possible les symétries ou les répétitions. Ce traitement du profil mélodique ne s'appuie sur aucune structure préexistante, empruntée ${ }^{15}$ : le fil du discours est géré par le personnage mot après mot, comme si c'était un monologue de théâtre parlé ou un récitatif dont on veut rehausser le taux lyrique (on pourrait presque considérer cette pièce comme l'accomplissement radical d'une esthétique de l'arioso plutôt que comme un numéro clos ordinaire). Expression sublime de logocentrisme lyrique, son chant avance sans se laisser dicter le chemin par des moules syntaxiques ou phraséologiques. Wagner, ennemi acharné de ce qu'il appelait la "Quadratur", adepte d'une ligne flexible qui garde l'équilibre interne et la continuité de la syntaxe mélodique sans s'aplatir sur des grilles métriques, affirmait justement avoir appris de Bellini l'art de la mélodie $^{16}$. En outre, Norma semble gérer la temporalité musicale à travers un rythme harmonique extrêmement étiré, dans lequel chaque fonction essentielle est prolongée à l'aide d'oscillations pendulaires internes de niveau hiérarchique inférieur (dominantes secondaires), donnant l'effet d'un temps immobilisé, l'illusion d'arrêt du flux du changement.

15. Aucune trace, par exemple, de ce qu'il est aujourd'hui convenu d'appeler «lyric form", la structure standard ААВA ou ААВC du bloc mélodique principal dans l'air d'opéra italien de cette période.

I6. Voir C. Wagner, Die Tagebücher, vol. III, p. 54 (7 mars 1878). 
Norma ne pourra rester longtemps ce qu'elle est au début: le profil inoubliable de ce personnage tient à ce que, à plusieurs moments, elle risque de basculer vers des modes émotionnels et des réactions qui la rendent plus normale, proche de nous. La friction entre ces différents modes d'expression se manifeste pour la première fois dans la scène qui précède le duo entre Norma et Adalgisa à la fin du premier acte, dans lequel Adalgisa raconte à Norma comment elle est tombée amoureuse d'un homme exceptionnellement beau aperçu dans l'enceinte du bois sacré. Ce récit évoque pour Norma le souvenir brûlant de sa rencontre, jadis, avec l'homme qu'elle a aimé (elles ignorent, pour l'instant, qu'il s'agit de la même personne). L'instant du souvenir serait approprié pour évoquer l'émotion de cette lointaine rencontre par un de ces motifs lyriques confiés à l'orchestre que l'opéra italien utilise pour sonoriser la remémoration intérieure. En effet (Ex. 1), la flûte énonce une émouvante mélodie élégiaque en $f a$ mineur, tandis que Norma prononce quelques mots de souvenir (O rimembranza! Io fui cosi rapita / al sol mirarlo in volto). Pour un instant, cette démarche active une focalisation musicale interne. Nous interprétons cette musique comme une plongée dans le souvenir de Norma, un instant d'effraction dans la psyché du personnage, typique d'une dramaturgie musicale moderne et "romantique». Toutefois, nous comprenons, dès qu'Adalgisa commence à chanter le tempo d'attacco du numéro (Sola, furtiva, al tempio), que cette mélodie de la flûte n'était que la ritournelle anticipant sa phrase vocale. L'ambiguïté est magnifiquement calculée: Norma a failli tomber dans un mode d'expression bourgeois/sentimental, en adoptant le matériau musical d'Adalgisa comme si c'était son propre souvenir. Cependant, cela ne dure qu'un instant: c'est bien à Adalgisa que cette musique se rattache. Nous ne connaîtrons jamais la teneur de la réminiscence, de l'image musicale qui habite la mémoire de Norma.

Une fois la trahison de Pollione apprise, Norma, accablée sous le poids du chagrin, semble céder et sa nature de personnage tragique entre en crise. Dans la grande scène nocturne du début du deuxième acte, dans laquelle elle frôle l'infanticide, l'expression verbale devient éparse, saccadée, dérangée: la dramaturgie fait appel à l'expression instrumentale, à la pantomime, aux gestes sonores qui trahissent les sursauts de la volonté. Les nombreuses didascalies annotées par Bellini dans la partition autographe - qui en général en contient assez peu - sont le signe de ce rapprochement provisoire d'une dramaturgie dans laquelle le gestuel, 

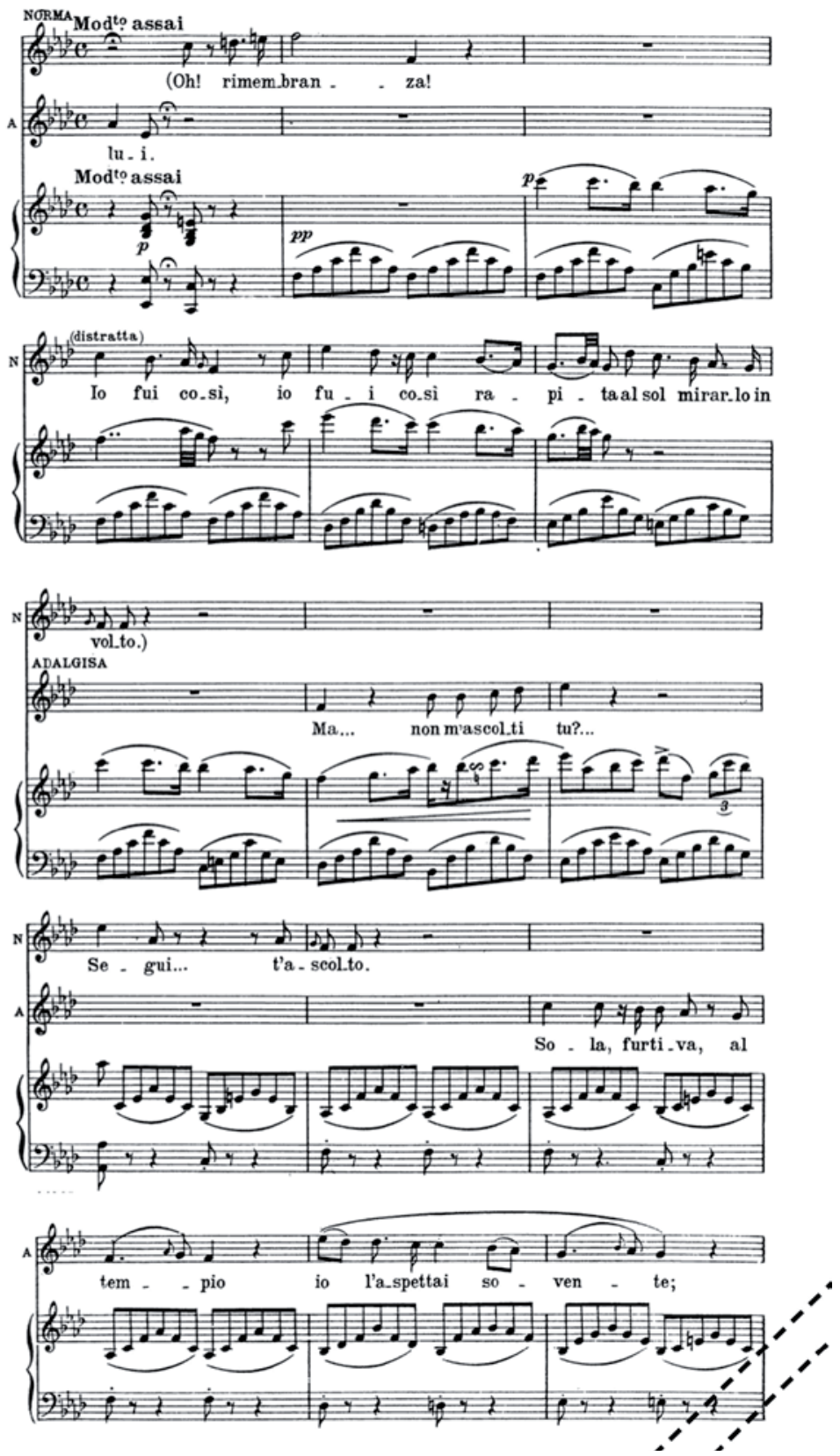

Ex. 1 - Norma, Acte I, O rimembranza! Io fui cosi rapita... 
le non-dit, les interventions narratives de l'orchestre prennent le dessus. On se croirait plongés dans une esthétique du drame ou du mélodrame, telle que l'avaient définie Diderot et Rousseau:

Qu'est-ce qui nous affecte dans le spectacle de l'homme animé de quelque grande passion? Sont-ce ses discours? Quelquefois. Mais ce qui émeut toujours, ce sont des cris, des mots inarticulés, des voix rompues $[\ldots . .]^{17}$.

L'acteur agité, transporté d'une passion qui ne lui permet pas de tout dire, s'interrompt, s'arrête, fait des réticences, durant lesquelles l'orchestre parle pour lui, et ces silences ainsi remplis affectent infiniment plus l'auditeur que si l'acteur disoit lui-même tout ce que la musique fait entendre ${ }^{18}$.

Toutefois, Norma, parvenue au bord du gouffre, surmonte la crise: par un geste cathartique inoubliable, c'est encore sa voix qui récupère, sur l'invocation teneri figli..., et sur le mode d'un arioso, la grande mélodie en ré mineur jouée par les violoncelles peu avant son entrée sur scène. C'est le début d'une remontée qui passe à travers les phases de la violence et du chantage jaloux (à remarquer l'assurance, la rigueur structurelle et phraséologique par laquelle Norma contrôle son dialogue avec Pollione prisonnier, In mia mano alfin tu sei) jusqu'au moment où elle accepte sa responsabilité. Et voici enfin le début de Qual cor tradisti, le passage cité par Schopenhauer (Ex. 2): trois mesures de simple accompagnement qui agissent comme des guillemets, comme une sorte de rideau qui rend au chant de Norma sa nature suprêmement artificielle et théâtrale, son caractère de haute transfiguration, sa nature libérée de toute contrainte matérielle et instinctuelle. L'ampleur et la structuration de la mélodie, le contrôle de tous les paramètres de l'écriture musicale montrent à quel point cette décision, l'acceptation de la mort, se situe dans les régions d'une nouvelle liberté, supérieure, de décision.

Il reste à régler le nœud très chargé émotionnellement du sort des enfants, que l'usage voudrait que l'on mette à mort puisqu'ils sont les fruits d'une liaison interdite et blasphématoire. On s'attendrait que l'imploration de Norma à Oroveso pour que leur vie soit sauvée surgisse de manière immédiate et décomposée, conformément à la représentation

I7. D. Diderot, Entretiens sur "Le Fils naturel», p. 1220.

I8. J.-J. Rousseau, Dictionnaire de musique, art. Récitatif obligé, p. 1013. 

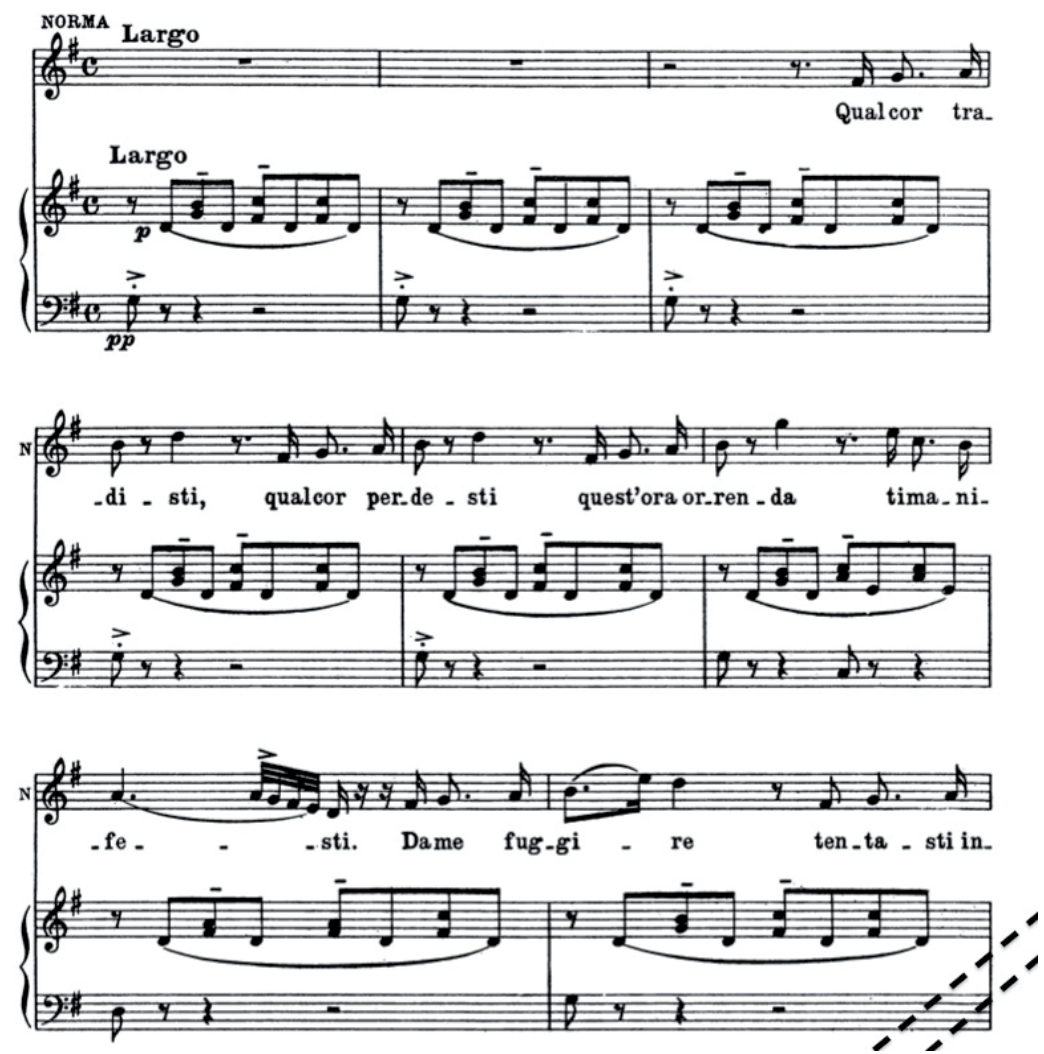

Ex. 2 - Norma, Acte II, Qual cor tradisti.

réaliste de l'angoisse mortelle d'une mère (qu'aurait pu en faire un Donizetti ?) mais il n'en est rien. La phrase de Norma, précédée par une mesure d'accompagnement qui fixe la tonalité de mi mineur et le profil plastique de la courte cellule d'accompagnement, démarre pour un parcours long et régulier de dix-huit mesures, Moderato. Par son chant très développé (une melodia lunga lunga, comme Verdi l'aurait appelée), qui donne l'impression de dominer tous les paramètres du discours musical à mesure qu'il se déroule, qu'il dépasse toute articulation syntaxique et qu'il conquiert les tessitures élevées, Norma déploie le souffle long et l'intensité d'une grande harangue tragique racinienne. Finalement, le plaidoyer débouche sur mi majeur: Oroveso semble céder et tous les personnages sur scène, y compris le chœur, s'unissent à ce grandiose concertato dont la culmination sur basse chromatique ascendante, jouée deux fois, est 
devenue une véritable icône pour l'opéra du XIXe siècle. Il est intéressant de remarquer que, sur le plan de la réalité scénique, les chœurs ne sont pas censés avoir entendu la prière de la protagoniste, ou du moins ils n'en ont pas été touchés, puisque, durant le concertato, ils chantent un texte qui confirme leur attitude de rejet féroce et leur soif barbare de vengeance:

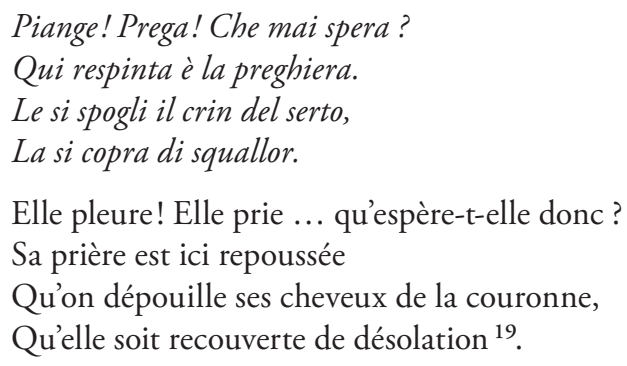

Rarement remarqués par les auditeurs, ces mots sont en décalage par rapport à la Stimmung de transfiguration qui saisit l'ensemble de l'écriture musicale durant ce concertato. Cette musique ne correspond pas à la réalité des choses: elle sonorise plutôt, comme un prisme subjectif qui transforme la perception de la réalité, l'aboutissement d'un processus de régénération intérieure et morale qui élève les protagonistes dans une dimension "autre», inatteignable. Sur le dernier accord du concertato, comme par une coupe cinématographique, le plan change: $m i$ mineur s'installe brusquement, le tempo devient Allegro agitato assai, le regard revient sur la réalité matérielle de la foule assoiffée de sang et sur la cérémonie de sacrifice humain qui va s'accomplir.

L'opéra italien des années 1810-1850 vit de sa nature ambiguë, de l'équilibre sans cesse renouvelé entre la tradition classique - perçue comme un élément identitaire de la culture nationale, mais également suspecte en tant qu'expression d'un passé aristocratique à surmonter - et le goût romantique. Dans Norma, Romani et Bellini parviennent à faire de ces deux tendances les pôles d'une dialectique dramaturgique et anthropologique, les ingrédients d'une synthèse qui aboutit, au bout d'un parcours tourmenté, à la récupération du modèle tragique et de sa vision du monde.

Luca Zoppelli Université de Fribourg

I9. Traduction d'Yseult Pelloso dans Bellini, Norma, p. 59. 


\section{BIBLIOGRAPHIE}

Balzac, Honoré de, La Comédie humaine, éd. publiée sous la direction de Pierre-Georges Castex, vol. I, Paris, Gallimard, 1976.

Bellini, Vincenzo, Norma, Paris, Ed. Premières Loges, 2007 (AvantScène Opéra, 236).

Cazaux, Chantal, «Alexandre Soumet, Felice Romani: d'une Norma l'autre», Musicorum, 5 (2006-2007), p. 269-286.

Cессні, Paolo, "Temi letterari e individuazione melodrammatica in Norma di Vincenzo Bellini ", Recercare, 9 (1997), p. 121-153.

Colas, Damien, "Aux sources du personnage de Norma», Bollettino di Studi Belliniani, 1 (2015), p. 5-37.

Diderot, Denis, Entretiens sur "Le Fils naturel", dans Euvres, éd. par E. A. Billy, Paris, Gallimard, 1951, p. 1201-1273.

Fumaroli, Marc, Héros et orateurs. Rhétorique et dramaturgie cornéliennes, Genève, Droz, 1990.

Gautier, Théophile, Histoire de l'art dramatique en France depuis vingtcinq ans, $4^{\mathrm{e}}$ série, Paris, Hetzel, 1859.

—, Histoire du romantisme, Paris, Charpentier, 1874.

Manzoni, Alessandro, Materiali estetici, in Scritti di estetica, a cura di Umberto Colombo, Milano, Edizioni Paoline, 1967, p. 469-516.

—, Adelchi, a cura di Sergio Blazina, Milano, Garzanti, 1991.

Melai, Maurizio, Les derniers feux de la tragédie classique au temps du romantisme, Paris, Presses de l'Université Paris-Sorbonne, 2015.

Neri, Carmelo, Vincenzo Bellini. Nuovo epistolario (1819-1835) con documenti inediti, Aci Sant'Antonio, Agorà, 2005.

Rousseau, Jean-Jacques, Dictionnaire de musique, art. Récitatif obligé, in Euvres complètes, t. 5. Ecrits sur la musique, la langue et le théâtre, Paris, Gallimard, 1995.

Schopenhauer, Arthur, Die Welt als Wille und Vorstellung, Leipzig, Brockhaus, 1859.

Wagner, Cosima, Die Tagebücher, hrsg. von Martin Gregor-Dellin, Dietrich Mack, München/Zürich, Piper, 1982. 
WAGNer, Richard, Bellini. Ein Wort zu seiner Zeit, in Musik-Konzepte 46: Vincenzo Bellini, hrsg. von H.-K. Metzger, R. Riehn, München, Text+Kritik, 1985.

Zoppelli, Luca, «Il personaggio belliniano. Poetica del drame e poetica tragica", in Vincenzo Bellini nel secondo centenario della nascita. Atti del Convegno Internazionale (Catania, novembre 2001), a cura di G. Seminara e A. Tedesco, Firenze, Olschki, 2004, p. 131-148. 
